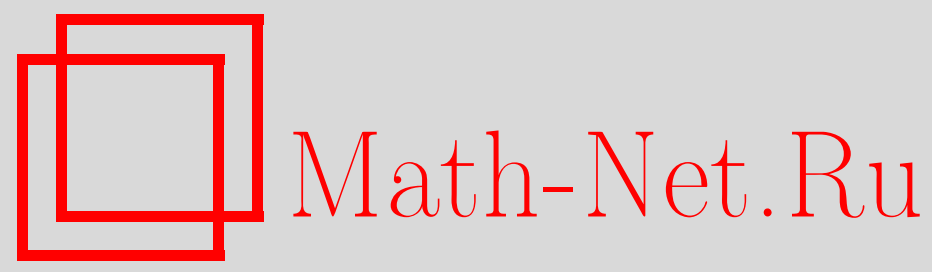

А. В. Булинский, Статистический вариант центральной предельной теоремы для векторных случайных полей, $M a$ тем. заметки, 2004, том 76, выпуск 4, 490-501

DOI: https://doi.org/10.4213/mzm123

Использование Общероссийского математического портала Math-Net.Ru подразумевает, что вы прочитали и согласны с пользовательским соглашением http://www . mathnet.ru/rus/agreement

Параметры загрузки:

IP : 107.22 .136 .117

26 апреля 2023 г., 15:14:22

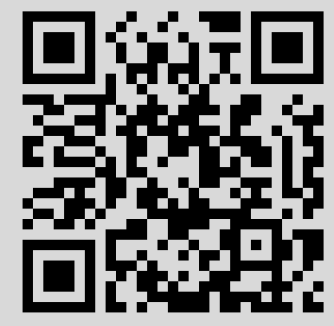




\section{СТАТИСТИЧЕСКИЙ ВАРИАНТ ЦЕНТРАЛЬНОЙ ПРЕДЕЛЬНОЙ ТЕОРЕМЫ ДЛЯ ВЕКТОРНЫХ СЛУЧАЙНЫХ ПОЛЕЙ}

\section{А. В. Булинский}

Классическая ЦПТ Ньюмена для действительных строго стационарных ассоциированных случайных полей обобщается на строго стационарные квази-ассоциированные векторно-значные случайные поля, охватываюшие, в частности, положительно или отрицательно ассоциированные поля с конечными вторыми моментами. Установлен также вариант ЦПТ со случайной матричной нормировкой, что позволяет строить приближенные доверительные интервалы для неизвестного вектора средних.

Библиография: 26 названий.

1. Введение. Цель этой работы - доказать центральную предельную теорему (ЦПТ), используя случайную матричную нормировку, для строго стационарных случайных полей, определенных на решетке $\mathbb{Z}^{d}$ и принимаюших значения в пространстве $\mathbb{R}^{k}$. Суммирование мультииндексированных случайных векторов ведется по конечным множествам $U_{n} \subset \mathbb{Z}^{d}$, в определенном смысле растущим при $n \rightarrow \infty$. Структура зависимости рассматриваемых полей описьвается в терминах квази-ассоииированности. Это понятие для действительных случайных полей было введено в [1] и для векторно-значных полей - в [2]. Родственные условия зависимости были предложены для случайных процессов в [3] и для полей - в [4].

Пусть $X=\left\{X_{t}, t \in T\right\}$ - семейство действительных случайных величин, определенных на вероятностном пространстве $(\Omega, \mathscr{F}, P)$ и индексированных переменной $t$ из некоторого множества $T$. Напомним, что это семейство назьвается ассоииированным или положительно зависимым [5], если для любых конечных множеств $I, J \subset T$ и всех покоординатно неубьвающих функций $f: \mathbb{R}^{|I|} \rightarrow \mathbb{R}, g: \mathbb{R}^{|J|} \rightarrow \mathbb{R}$

$$
\operatorname{cov}\left(f\left(X_{s}, s \in I\right), g\left(X_{t}, t \in J\right)\right) \geqslant 0
$$

когда эта ковариация существует (здесь и далее $|I|$ обозначает число элементов конечного множества $I)$. Запись $f\left(X_{s}, s \in I\right)$ означает, что для $I=\left\{s_{1}, \ldots, s_{m}\right\}$ рассматривается произвольная функция $f\left(X_{u_{1}}, \ldots, X_{u_{m}}\right)$, где $\left(u_{1}, \ldots, u_{m}\right)$ - любая перестановка набора $\left(s_{1}, \ldots, s_{m}\right)$.

Работа выполнена при частичной поддержке Российского фонда фундаментальных исследований, грант № 01-03-00724, программы “Ведущие научные школы”, грант № НШ-1758.2003.1, и фонда INTAS, грант № 03-51-5018. 
Имеются различные модификации этого определения. При дополнительном условии $I \cap J=\varnothing$ соотношение (1) вводит слабую ассоциированность [6] или положительную ассоииированность, а аналог (1) с неравенством противоположного знака задает отрицательную ассоииированность [7].

Заметим, что любое семейство независимых действительных случайных величин автоматически является ассоциированньм и отрицательно ассоциированным.

В математической статистике, теории надежности, теории перколяции, статистической физике и самой теории вероятностей существуют интересные стохастические модели, описьваемые семействами положительно или отрицательно ассоциированных случайных величин (см., например, [5]-[14]). В физической литературе, как правило, используются "FKG-неравенства", влекущие ассоциированность.

Набор действительных случайных величин $X=\left\{X_{t}, t \in T\right\}$, имеющих $\mathrm{E} X_{t}^{2}<\infty$, $t \in T$, назьвается квази-ассоциированным [1], если для всех конечных непересекающихся множеств $I, J \subset T$ и любых ограниченных липшицевых функций $f: \mathbb{R}^{|I|} \rightarrow \mathbb{R}$, $g: \mathbb{R}^{|J|} \rightarrow \mathbb{R}$ вьполняется следующее неравенство:

$$
\left|\operatorname{cov}\left(f\left(X_{s}, s \in I\right), g\left(X_{t}, t \in J\right)\right)\right| \leqslant \operatorname{Lip}(f) \operatorname{Lip}(g) \sum_{s \in I} \sum_{t \in J}\left|\operatorname{cov}\left(X_{s}, X_{t}\right)\right| .
$$

Здесь

$$
\operatorname{Lip}(f)=\sup _{x \neq y} \frac{|f(x)-f(y)|}{\|x-y\|_{1}}<\infty,
$$

$\|x\|_{1}=\sum_{s=1}^{m}\left|x_{s}\right|$ для $x=\left(x_{1}, \ldots, x_{m}\right) \in \mathbb{R}^{m}$. Поскольку все нормы в пространстве $\mathbb{R}^{m}$ эквивалентны, выбор нормы $\|\cdot\|_{1}$ в (3) произведен лишь для удобства. Разумеется, в правую часть определения (2) можно включить и дополнительные множители.

В [15] было показано, что любое семейство положительно или отрицательно ассоциированных случайных величин с конечными вторыми моментами удовлетворяет неравенству (2). Следовательно, такие случайные поля всегда квази-ассоциированны.

Аналог (2) в случае гладких функций $f$ и $g$ был установлен в [16] для ассоциированных случайных величин (cp. [17]-[19]).

Пусть теперь $X=\left\{X_{t}, t \in T\right\}-$ случайное поле со значениями в пространстве $\mathbb{R}^{k}$. Обобщения упомянутых вьше условий зависимости на семейства векторно-значньх случайных величин рассматривались, например, в [20], [2] и [21]. Следующее определение дано в [2].

Случайное поле $X$ со значениями в $\mathbb{R}^{k}$ назьвается квази-ассочиированным, если для любых непересекающихся множеств $I, J \subset \mathbb{Z}^{d}$ и всех ограниченных липшицевых функций $f: \mathbb{R}^{k|I|} \rightarrow \mathbb{R}, g: \mathbb{R}^{k|J|} \rightarrow \mathbb{R}$ имеет место неравенство

$$
\left|\operatorname{cov}\left(f\left(X_{s}, s \in I\right), g\left(X_{t}, t \in J\right)\right)\right| \leqslant \operatorname{Lip}(f) \operatorname{Lip}(g) \sum_{s \in I} \sum_{t \in J} \sum_{r, q=1}^{k}\left|\operatorname{cov}\left(X_{s, r}, X_{t, q}\right)\right|,
$$

где $X_{s, r}$ обозначает $r$-ю компоненту вектора $X_{s}$.

Недавно в [22] было доказано, что любоегауссовское случайноеполе $X=\left\{X_{t}, t \in T\right\}$ со значениями в $\mathbb{R}^{k}$ является квази-ассоциированньм. Действительноегауссовское случайное поле $X$ обладает свойством ассоциированности тогда и только тогда (см. [13]), когда его ковариационная функция неотрищательна, и обладает свойством отрицательной ассоциированности тогда и только тогда (см. [7]), когда $\operatorname{cov}\left(X_{s}, X_{t}\right) \leqslant 0$ при всех 
$s \neq t$. Таким образом, результаты [15] и [22] показьвают, что понятие квази-ассоциированности приводит для полей с конечными вторьми моментами к строго болееширокому классу объектов, чем понятия положительной и отрицательной ассоциированности.

Заканчивая обсуждение условий зависимости, заметим, что имеются различные возможности давать оценки левой части неравенства (4) для тех или иных классов “пробных функций" $f$ и $g$ (см., например, [23], [4] и [21]).

В п. 2 данной работы классическая теорема Ньюмена [12] обобщается на векторно-значные строго стационарные квази-ассоциированные случайные поля. В п. 3 доказьвается статистический вариант ЦПТ. Для этого используются самонормализованные частные суммы случайных векторов.

2. ЦПТ для квази-ассоциированных строго стационарных векторных полей. В этом пункте мы докажем ЦПТ для частных сумм

$$
S\left(U_{n}\right)=\sum_{j \in U_{n}} X_{j}, \quad n \in \mathbb{N},
$$

мультииндексированных квази-ассоциированных случайных векторов $X_{j}$, используя неслучайную нормировку. Суммирование ведется по конечньм множествам $U_{n} \subset \mathbb{Z}^{d}$, растущим в должном смысле.

$$
\begin{gathered}
\text { Для } a=\left(a_{1}, \ldots, a_{d}\right) \in \mathbb{R}_{+}^{d}, V \subset \mathbb{R}^{d} \text { и } j=\left(j_{1}, \ldots, j_{d}\right) \in \mathbb{Z}^{d} \text { пусть } \\
\Lambda_{0}(a)=\left\{x=\left(x_{1}, \ldots, x_{d}\right): 0<x_{p} \leqslant a_{p}, p=1, \ldots, d\right\}, \\
\Lambda_{j}(a)=\Lambda_{0}(a)+\left(j_{1} a_{1}, \ldots, j_{d} a_{d}\right), \\
J_{a}^{+}(V)=\left\{j: \Lambda_{j}(a) \cap V \neq \varnothing\right\}, \quad N_{a}^{+}(V)=\left|J_{a}^{+}(V)\right|, \\
J_{a}^{-}(V)=\left\{j: \Lambda_{j}(a) \subset V\right\}, \quad N_{a}^{-}(V)=\left|J_{a}^{-}(V)\right| .
\end{gathered}
$$

Говорят, что $V_{n} \rightarrow \infty$ в смысле Ван Хова при $n \rightarrow \infty$, если для каждого $a \in \mathbb{R}_{+}^{d}$

$$
N_{a}^{-}\left(V_{n}\right) \rightarrow \infty \text { и } \frac{N_{a}^{-}\left(V_{n}\right)}{N_{a}^{+}\left(V_{n}\right)} \rightarrow 1 \text { при } n \rightarrow \infty .
$$

Если $V_{n}$ - ограниченные измеримые подмножества $\mathbb{R}^{d}$, то (см. [24, гл. $\left.\left.2, \S 2.1\right]\right) V_{n} \rightarrow \infty$ в смысле Ван Хова тогда и только тогда, когда для любого $\varepsilon>0$ верно соотношение

$$
\frac{\mu\left(\left(\partial V_{n}\right)^{\varepsilon}\right)}{\mu\left(V_{n}\right)} \rightarrow 0 \quad \text { при } n \rightarrow \infty,
$$

где $G^{\varepsilon}$ обозначает $\varepsilon$-окрестность (в евклидовой метрике) множества $G \subset \mathbb{R}^{d}$ и $\mu$-мера Лебега в $\mathbb{R}^{d}$.

Естественньй дискретный аналог этого понятия "регулярного роста" множеств $U_{n} \subset \mathbb{Z}^{d}$ был использован, например, в [25]. Для $U \subset \mathbb{Z}^{d}$ положим

$$
\partial U=\left\{s \in U: \inf _{t \in \mathbb{Z}^{d} \backslash U}\|s-t\|=1\right\},
$$

где $\|x\|=\max _{1 \leqslant p \leqslant d}\left|x_{p}\right|, x=\left(x_{1}, \ldots, x_{d}\right) \in \mathbb{R}^{d}$.

Говорят, что последовательность $\left\{U_{n}\right\}_{n \in \mathbb{N}}$ конечных подмножеств $\mathbb{Z}^{d}$ стремится к бесконечности регулярным образом (ср. с (10)), если

$$
\frac{\left|\partial U_{n}\right|}{\left|U_{n}\right|} \rightarrow 0 \quad \text { при } n \rightarrow \infty \text {. }
$$

Следующий результат является обобщением теоремы 1 из [26], которая, в свою очередь, расширяла область действия классической ЦПТ Ньюмена [12]. 
Теорема 1. Пусть $X=\left\{X_{j}, j \in \mathbb{Z}^{d}\right\}$ - строго стационарное квази-ассоциированное случайное поле со значениями в пространстве $\mathbb{R}^{k}$. Предположим, что при всех $r, q=1, \ldots, k$

$$
\sigma_{r, q}=\sum_{j \in \mathbb{Z}^{d}}\left|\operatorname{cov}\left(X_{0, r}, X_{j, q}\right)\right|<\infty
$$

Тогда для любых конечных множеств $U_{n} \subset \mathbb{Z}^{d}$, удовлетворяющих условию (11), справедливо соотношение

$$
\left|U_{n}\right|^{-1 / 2}\left(S\left(U_{n}\right)-\left|U_{n}\right| \mathrm{E} X_{0}\right) \stackrel{D}{\rightarrow} N(0, C) \quad n p u \quad n \rightarrow \infty
$$

Здесь $C$ - матрица с әлементами

$$
c_{r, q}=\sum_{j \in \mathbb{Z}^{d}} \operatorname{cov}\left(X_{0, r}, X_{j, q}\right), \quad r, q=1, \ldots, k
$$

$u$ “ $\stackrel{D}{\rightarrow}$ " обозначает слабую сходимость распределений векторов в $\mathbb{R}^{k}$.

ДокАЗАТЕЛЬСТВо этой теоремы основано на известных методах (см. [12], [20] и [26]). Начнем с вспомогательных результатов.

Лемма 1. Пусть $X=\left\{X_{j}, j \in \mathbb{Z}^{d}\right\}$ - стационарное в иироком смысле случайное поле со значениями в $\mathbb{R}^{k}$ такое, что выполнено условие $(12)$. Пусть $\left\{U_{n}\right\}_{n \in \mathbb{N}}$ - последовательность регулярно растущих конечных подмнохсеств $\mathbb{Z}^{d}($ см. (11)). Тогда

$$
\left|U_{n}\right|^{-1} \operatorname{var} S\left(U_{n}\right) \rightarrow C \quad n p u \quad n \rightarrow \infty
$$

əде $\operatorname{var} S\left(U_{n}\right)$ - ковариационная матрица $S\left(U_{n}\right)$ и $C$ - матрица, введенная согласно (14). Соотношение (15) означает сходимость всех әлементов матрии, $\left|U_{n}\right|^{-1} \operatorname{var} S_{n} \kappa$ соответствуюшим әлементам матрицы $C$ при $n \rightarrow \infty$.

Для строго стационарного действительного случайного поля $X$ (т.е. при $k=1$ ) это утверждение было установлено в работе [25]. В многомерном случае для полей, стационарных в широком смысле, доказательство полностью аналогично.

Следующая лемма представляет собой расширение сферы действия неравенства, доказанного в статье [20].

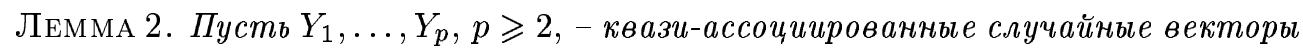
со значениями в $\mathbb{R}^{k}$. Тогда для любого $\lambda \in \mathbb{R}^{k}$ имеет место оченка

$$
\left|\mathrm{E} \exp \left\{i \sum_{m=1}^{p}\left(\lambda, Y_{m}\right)\right\}-\prod_{m=1}^{p} \mathrm{E} \exp \left\{i\left(\lambda, Y_{m}\right)\right\}\right| \leqslant \sqrt{2}\|\lambda\|^{2} \sum_{\substack{m, s=1 \\ m \neq s}}^{p} \sum_{r, q=1}^{k}\left|\operatorname{cov}\left(Y_{m, r}, Y_{s, q}\right)\right|
$$

әде $(\cdot, \cdot)$ обозначает скалярное произведение в $\mathbb{R}^{k}$ и $i^{2}=-1$. 
ДокАЗАТЕЛЬСТво. Для всех $\lambda \in \mathbb{R}^{k}$ и $1 \leqslant v \leqslant p-1, v \in \mathbb{N}$, верно тождество

$$
\begin{aligned}
\Delta_{v, p}= & \mathrm{E} \exp \left\{i \sum_{u=v}^{p}\left(\lambda, Y_{u}\right)\right\}-\mathrm{E} \exp \left\{i\left(\lambda, Y_{v}\right)\right\} \mathrm{E} \exp \left\{i \sum_{u=v+1}^{p}\left(\lambda, Y_{u}\right)\right\} \\
= & \left.\operatorname{cov}\left(\cos \left(\lambda, Y_{v}\right), \cos \left(\sum_{u=v+1}^{p}\left(\lambda, Y_{u}\right)\right)\right)\right)-\operatorname{cov}\left(\sin \left(\lambda, Y_{v}\right), \sin \left(\sum_{u=v+1}^{p}\left(\lambda, Y_{u}\right)\right)\right) \\
& +i\left[\operatorname{cov}\left(\cos \left(\lambda, Y_{v}\right), \sin \left(\sum_{u=v+1}^{p}\left(\lambda, Y_{u}\right)\right)\right)\right. \\
& \left.+\operatorname{cov}\left(\sin \left(\lambda, Y_{v}\right), \cos \left(\sum_{u=v+1}^{p}\left(\lambda, Y_{u}\right)\right)\right)\right] .
\end{aligned}
$$

Заметим, что $f\left(x_{1}, \ldots, x_{k}\right)=\cos \left(\sum_{r=1}^{k} \lambda_{r} x_{r}\right)$ и

$$
g\left(x_{v+1,1}, \ldots, x_{v+1, k}, \ldots, x_{p, 1}, \ldots, x_{p, k}\right)=\cos \left(\sum_{r=1}^{k} \lambda_{r} \sum_{q=v+1}^{p} x_{q, r}\right)
$$

при каждом фиксированном $\lambda \in \mathbb{R}^{k}$ являются липшицевыми функциями такими, что $\operatorname{Lip}(f) \leqslant\|\lambda\|$ и $\operatorname{Lip}(g) \leqslant\|\lambda\|$. Используя аналогичные неравенства для функций типа синусов, а также тривиальную оценку $|a+i b| \leqslant \sqrt{2} \max \{|a|,|b|\}$, где $a, b \in \mathbb{R}$, мы видим, что квази-ассоциированность набора величин $Y_{1}, \ldots, Y_{p}$ влечет неравенство

$$
\left|\Delta_{v, p}\right| \leqslant 2 \sqrt{2}|| \lambda \|^{2} \sum_{u=v+1}^{p} \sum_{r, t=1}^{k}\left|\operatorname{cov}\left(Y_{v, r}, Y_{u, t}\right)\right| .
$$

Левую часть (16) оценивает $\sum_{v=1}^{p-1}\left|\Delta_{v, p}\right|$. Это завершает доказательство леммы.

Теперь перейдем к доказательству теоремы 1 . Для $a=\left(a_{1}, \ldots, a_{d}\right) \in \mathbb{R}_{+}^{d}, U \subset \mathbb{Z}^{d}$ и $j \in \mathbb{Z}^{d}$ положим $\widetilde{\Lambda}_{0}(a)=\Lambda_{0}(a) \cap \mathbb{Z}^{d}, \widetilde{\Lambda}_{j}(a)=\Lambda_{j}(a) \cap \mathbb{Z}^{d}$, где $\Lambda_{0}(a)$ и $\Lambda_{j}(a)$ были введены в (5) и (6). Тогда для $U \subset \mathbb{Z}^{d}$ точно так же, как для $V \subset \mathbb{R}^{d}$ (т.е. используя $\widetilde{\Lambda}_{0}(a)$ и $\widetilde{\Lambda}_{j}(a)$ вместо $\Lambda_{0}(a)$ и $\Lambda_{j}(a)$ в $\left.(7),(8)\right)$ определим $J_{a}^{+}(U)$ и $J_{a}^{-}(U)$.

Для каждого фиксированного $a \in \mathbb{R}_{+}^{d}$ рассмотрим множество $M_{n}=J_{a}^{-}\left(U_{n}\right)$ и

$$
U_{n}^{(1)}=\bigcup_{j \in M_{n}} \widetilde{\Lambda}_{j}(a), \quad U_{n}^{(0)}=U_{n} \backslash U_{n}^{(1)}, n \in \mathbb{N} .
$$

Пусть $\|\cdot\|_{0}-$ евклидова норма в $\mathbb{R}^{k}$. Имеем

$$
\mathrm{E}\left\|\sum_{j \in U_{n}^{(0)}}\left(X_{j}-\mathrm{E} X_{j}\right)\right\|_{0}^{2} \leqslant\left|U_{n}^{(0)}\right| \sum_{r=1}^{k} \sigma_{r, r} .
$$

Поэтому легко видеть, что в силу справедливости соотношений

$$
\frac{\left|U_{n}^{(0)}\right|}{\left|U_{n}\right|} \rightarrow 0 \text { и } \frac{\left|U_{n}^{(1)}\right|}{\left|U_{n}\right|} \rightarrow 1 \quad \text { при } n \rightarrow \infty
$$


для доказательства (13) достаточно установить следующее:

$$
\left|U_{n}^{(1)}\right|^{-1 / 2}\left(S\left(U_{n}^{(1)}\right)-\left|U_{n}^{(1)}\right| \mathrm{E} X_{0}\right) \stackrel{D}{\rightarrow} N(0, C(a)), \quad n \rightarrow \infty
$$

где $C(a) \rightarrow C$ при $a \rightarrow \infty$ (т.е. $a_{r} \rightarrow \infty$ для каждого $\left.r=1, \ldots, k\right)$.

Введем случайные поля $\left\{Y_{j}(a), j \in M_{n}\right\}, n \in \mathbb{N}$, где

$$
Y_{j}(a)=\left|\widetilde{\Lambda}_{j}(a)\right|^{-1 / 2}\left(S\left(\widetilde{\Lambda}_{j}(a)\right)-\left|\widetilde{\Lambda}_{j}(a)\right| \mathrm{E} X_{0}\right) .
$$

Тогда

$$
\left|U_{n}^{(1)}\right|^{-1 / 2}\left(S\left(U_{n}^{(1)}\right)-\left|U_{n}^{(1)}\right| \mathrm{E} X_{0}\right)=\left|M_{n}\right|^{-1 / 2} \sum_{j \in M_{n}} Y_{j}(a) .
$$

При всех $j \in \mathbb{Z}^{d}$ по лемме 1 имеем

$$
\operatorname{var} Y_{j}(a)=\frac{\operatorname{var} S\left(\widetilde{\Lambda}_{0}(a)\right)}{\left|\widetilde{\Lambda}_{0}(a)\right|}=C(a) \rightarrow C \quad \text { при } a \rightarrow \infty
$$

Очевидно, $\left\{\left|M_{n}\right|^{-1 / 2} Y_{j}(a), j \in M_{n}\right\}$ для каждого $n \in \mathbb{N}$ и $a \in \mathbb{R}_{+}^{k}$ является набором квази-ассоциированных случайных векторов. Следовательно, лемма 2 и (23) позволят свести доказательство (20) к ЦПТ для массива независимых центрированных случайных векторов, имеющих ковариационную матрицу $C(a)$, если при любом $a \in \mathbb{R}_{+}^{k}$

$$
\Delta(n, a)=\frac{1}{\left|M_{n}\right|} \sum_{\substack{s, t \in M_{n} \\ s \neq t}} \sum_{r, q=1}^{k}\left|\operatorname{cov}\left(Y_{s, r}(a), Y_{t, q}(a)\right)\right| \rightarrow 0 \quad \text { при } n \rightarrow \infty .
$$

Для $\varepsilon \in(0,1 / 2)$ рассмотрим множества

$$
\Lambda_{0}^{\varepsilon}(a)=\left\{s \in \mathbb{Z}^{d}: \varepsilon a_{r}<s_{r} \leqslant(1-\varepsilon) a_{r}, r=1, \ldots, k\right\}
$$

Пусть $S_{r}(U)$ обозначает $r$-ю компоненту вектора $S(U), U \subset \mathbb{Z}^{d}$. Тогда при всех $n \in \mathbb{N}$ и $a \in \mathbb{R}_{+}^{d}$

$$
\begin{aligned}
\Delta(n, a) & \leqslant\left|\Lambda_{0}(a)\right|^{-1} \sum_{j \neq 0} \sum_{r, q=1}^{k}\left|\operatorname{cov}\left(S_{r}\left(\Lambda_{0}(a)\right), S_{q}\left(\Lambda_{j}(a)\right)\right)\right| \\
& \leqslant \sum_{\|j\|>\varepsilon a_{0}} \sum_{r, q=1}^{k}\left|\operatorname{cov}\left(X_{0, r}, X_{j, q}\right)\right|+\left(1-\frac{\left|\Lambda_{0}^{\varepsilon}(a)\right|}{\left|\Lambda_{0}(a)\right|}\right) \sum_{r, q=1}^{k} \sigma_{r, q},
\end{aligned}
$$

где $a_{0}=\min _{r=1, \ldots, k}\left|a_{r}\right|$. Выберем $\varepsilon$ достаточно малым, а затем возьмем $a \in \mathbb{R}_{+}^{d}$ достаточно большим (т.е. все компоненты $a$ будут достаточно велики). В силу (12) мы получим из (24) требуемый результат. Доказательство завершено. 
ЗАмЕчАниЕ 1. Мы дали детальное доказательство теоремы 1, чтобы выявить роль условия квази-ассоциированности. Более того, как видно, чтобы доказать ЦПТ для строго стационарных векторно-значных полей, достаточно использовать в (4) должные оценки ковариаций только функций типа синусов и косинусов от определенным образом нормализованных сумм исходных случайных векторов, берушихся по кубам. Конечно, при этом налагаются условия вида (11) и (12).

ЗАмечание 2. Так же, как в работе [12], можно использовать ренорм-группы для построения полей типа (21) при всех $j \in \mathbb{Z}^{d}$. Рассуждения, проведенныепри доказательстве теоремы 1 , показывают, что конечномерные распределения таких полей с ростом параллелепипедов разбиения $\mathbb{R}^{d}$ будут слабо сходиться к соответствующим конечномерным распределениям центрированного гауссовского поля, имеющего независимые значения.

3. Статистический вариант ЦПТ. Чтобы построить приближенные доверительные области для неизвестного вектора средних строго стационарного квази-ассоциированного случайного поля со значениями в $\mathbb{R}^{k}$, требуются состоятельные оценки ковариационной матрицы $C$, введенной в (13). Если $C$ - невырожденная матрица, то (13) влечет следующее соотношение:

$$
\left(C\left|U_{n}\right|\right)^{-1 / 2}\left(S\left(U_{n}\right)-\left|U_{n}\right| \mathrm{E} X_{0}\right) \stackrel{D}{\rightarrow} N(0, I),
$$

где $I$ - единичная матрица порядка $k$. Таким образом, если имеется последовательность состоятельных оценок $\widehat{C}\left(U_{n}\right)=\left(\widehat{c}_{r, q}\left(U_{n}\right)\right)_{r, q=1}^{k}$ матрицы $C=\left(c_{r, q}\right)_{r, q=1}^{k}$, т.е. таких, что при всех $r, q=1, \ldots, k$

$$
\widehat{c}_{r, q}\left(U_{n}\right) \stackrel{P}{\rightarrow} c_{r, q} \quad \text { при } n \rightarrow \infty,
$$

то в силу (25) и (26) приходим к формуле

$$
\left(\widehat{C}\left(U_{n}\right)\left|U_{n}\right|\right)^{-1 / 2}\left(S\left(U_{n}\right)-\left|U_{n}\right| \mathrm{E} X_{0}\right) \stackrel{D}{\rightarrow} N(0, I) .
$$

Здесь $\widehat{C}\left(U_{n}\right)=\widehat{C}\left(X_{j}, j \in U_{n}\right), n \in \mathbb{N}$, и “ $\stackrel{P}{\rightarrow}$ ” обозначает сходимость по вероятности.

Другими словами, в ЦПТ используется случайная нормализация. В этой связи можно напомнить известную процедуру стьюдентизации для независимых слагаемых.

Для строго стационарной последовательности $(d=1)$, обладающей перемешиванием или свойством ассоциированности, и множеств $U_{n}=\{1, \ldots, n\}, n \in \mathbb{N}$, две оценки дисперсий частных сумм были предложены в статье [18]. Эти оценки обеспечивали ЦПТ со случайной нормировкой. Более общие семейства оценок дисперсий частных сумм были введены в [26]. В последней работе изучались действительные ассоциированные случайные поля.

$$
\begin{array}{r}
\text { Для } j \in U \subset \mathbb{Z}^{d}(1 \leqslant|U|<\infty), b=b(U)>0 \text { и } r, q=1, \ldots, k \text { положим } \\
K_{j}(b)=\left\{t \in \mathbb{Z}^{d}:\|j-t\| \leqslant b\right\}, \quad Q_{j}=Q_{j}(U, b)=U \cap K_{j}(b), \\
\widehat{c}_{r, q}(U)=\frac{1}{|U|} \sum_{j \in U}\left|Q_{j}\right|\left(\frac{S_{r}\left(Q_{j}\right)}{\left|Q_{j}\right|}-\frac{S_{r}(U)}{|U|}\right)\left(\frac{S_{q}\left(Q_{j}\right)}{\left|Q_{j}\right|}-\frac{S_{q}(U)}{|U|}\right) .
\end{array}
$$

Заметим, что для зависимых слагаемых вместо традиционных оценок ковариационной матрицы, применяемых в случае независимых наблюдений, здесь возникают усредненные величины $S_{r}\left(Q_{j}\right) /\left|Q_{j}\right|$. 
Теорема 2. Пусть выполнены условия теоремы 1. Пусть $\left\{U_{n}\right\}_{n \in \mathbb{N}}-$ последовательность регулярно растущих конечных множеств $U_{n} \subset \mathbb{Z}^{d}$ (т.е. удовлетворяющих (11)). Предположим, что $b\left(U_{n}\right)=b_{n}$, где $\left\{b_{n}\right\}_{n \in \mathbb{N}}-$ такая последовательность натуральных чисел, что

$$
b_{n} \rightarrow \infty, \quad \frac{b_{n}^{d}\left|\partial U_{n}\right|}{\left|U_{n}\right|} \rightarrow 0, \quad \frac{b_{n}^{3 d}\left|\partial U_{n}\right|}{\left|U_{n}\right|^{2}} \rightarrow 0 \quad \text { npu } \quad n \rightarrow \infty .
$$

Тогда справедливо соотношение (26). Кроме того, если матрица С невырожденная, тогда имеет место (27).

ДокаЗАТЕльСтво. Оценки $\left(\widehat{c}_{r, q}(U)\right)_{r, q=1}^{k}$, введенные согласно (29), а также элементы матрицы $C$ инвариантны относительно преобразования $X_{j} \mapsto X_{j}-\mathrm{E} X_{0}, j \in U$. Поэтому без потери общности мы можем далее считать, что $\mathrm{E} X_{0}=0 \in \mathbb{R}^{k}$. Обозначим $\|\xi\|_{L}$ норму случайной величины $\xi$ в пространстве $L=L^{1}(\Omega, \mathscr{F}, P)$. Для каждых фиксированных $r, q=1, \ldots, k$ имеем

$$
\left\|\widehat{c}_{r, q}\left(U_{n}\right)-c_{r, q}\right\|_{L} \leqslant I_{1}\left(U_{n}\right)+I_{2}\left(U_{n}\right)+I_{3}\left(U_{n}\right),
$$

где

$$
\begin{aligned}
I_{1}\left(U_{n}\right)= & \frac{1}{\left|U_{n}\right|} \| \sum_{j \in U_{n}}\left|Q_{j}\right|\left\{\left(\frac{S_{r}\left(Q_{j}\right)}{\left|Q_{j}\right|}-\frac{S_{r}\left(U_{n}\right)}{\left|U_{n}\right|}\right)\left(\frac{S_{q}\left(Q_{j}\right)}{\left|Q_{j}\right|}-\frac{S_{q}\left(U_{n}\right)}{\left|U_{n}\right|}\right)\right. \\
& \left.-\frac{S_{r}\left(Q_{j}\right)}{\left|Q_{j}\right|} \frac{S_{q}\left(Q_{j}\right)}{\left|Q_{j}\right|}\right\} \|_{L}, \\
I_{2}\left(U_{n}\right)= & \frac{1}{\left|U_{n}\right|}\left\|\sum_{j \in U_{n}} \frac{1}{\left|Q_{j}\right|}\left(S_{r}\left(Q_{j}\right) S_{q}\left(Q_{j}\right)-\mathrm{E} S_{r}\left(Q_{j}\right) S_{q}\left(Q_{j}\right)\right)\right\|_{L}, \\
I_{3}\left(U_{n}\right)= & \left|\frac{1}{\left|U_{n}\right|} \sum_{j \in U_{n}} \frac{1}{\left|Q_{j}\right|} \mathrm{E} S_{r}\left(Q_{j}\right) S_{q}\left(Q_{j}\right)-c_{r, q}\right| .
\end{aligned}
$$

В силу условий (12) и (30)

$$
\begin{aligned}
I_{1}\left(U_{n}\right) \leqslant & \left|U_{n}\right|^{-3} \mathrm{E}\left|S_{r}\left(U_{n}\right) S_{q}\left(U_{n}\right)\right| \sum_{j \in U_{n}}\left|Q_{j}\right| \\
& +\left|U_{n}\right|^{-2} \sum_{j \in U_{n}}\left(\mathrm{E}\left|S_{r}\left(Q_{j}\right) S_{q}\left(U_{n}\right)\right|+\mathrm{E}\left|S_{r}\left(U_{n}\right) S_{q}\left(Q_{j}\right)\right|\right) \\
\leqslant & \left(\sigma_{r, r} \sigma_{q, q}\right)^{1 / 2}\left\{\left|K_{0}\left(b_{n}\right)\right|\left|U_{n}\right|^{-1}+2\left|K_{0}\left(b_{n}\right)\right|^{1 / 2}\left|U_{n}\right|^{-1 / 2}\right\} \rightarrow 0 \quad \text { при } n \rightarrow \infty .
\end{aligned}
$$

Для $c>0$ введем функции

$$
h_{1}(x)=\operatorname{sign}(x) \min \{|x|, c\}, \quad h_{2}(x)=x-h_{1}(x), \quad x \in \mathbb{R} .
$$

Для непустого конечного множества $Q \subset \mathbb{Z}^{d}$ положим

$$
\bar{S}_{r}(Q)=\frac{S_{r}(Q)}{\sqrt{|Q|}}, \quad r=1, \ldots, k .
$$


Заметим, что

$$
I_{2}\left(U_{n}\right) \leqslant \sum_{p, m=1}^{2} I_{2}^{(p, m)}\left(U_{n}\right)
$$

где

$$
I_{2}^{(p, m)}\left(U_{n}\right)=\frac{1}{\left|U_{n}\right|}\left\|\sum_{j \in U_{n}} h_{p}\left(\bar{S}_{r}\left(Q_{j}\right)\right) h_{m}\left(\bar{S}_{q}\left(Q_{j}\right)\right)-\mathrm{E} h_{p}\left(\bar{S}_{r}\left(Q_{j}\right)\right) h_{m}\left(\bar{S}_{q}\left(Q_{j}\right)\right)\right\|_{L} .
$$

Для $b, n \in \mathbb{N}$ определим множества

$$
T_{n}^{(b)}=\left\{s \in U_{n}: \inf _{t \in \partial U_{n}}\|s-t\| \leqslant b\right\} .
$$

Введем $T_{n}=T_{n}^{\left(b_{n}\right)}$, где $b_{n}$ удовлетворяют условию (30). Тогда

$$
\begin{aligned}
& I_{2}^{(1,2)}\left(U_{n}\right)+I_{2}^{(2,1)}\left(U_{n}\right)+I_{2}^{(2,2)}\left(U_{n}\right) \\
& \leqslant 2\left|U_{n}\right|^{-1} \sum_{j \in U_{n}}\left(\mathrm{E}\left|h_{1}\left(\bar{S}_{r}\left(Q_{j}\right)\right) h_{2}\left(\bar{S}_{q}\left(Q_{j}\right)\right)\right|+\mathrm{E}\left|h_{2}\left(\bar{S}_{r}\left(Q_{j}\right)\right) h_{1}\left(\bar{S}_{q}\left(Q_{j}\right)\right)\right|\right. \\
&\left.+\mathrm{E}\left|h_{2}\left(\bar{S}_{r}\left(Q_{j}\right)\right) h_{2}\left(\bar{S}_{q}\left(Q_{j}\right)\right)\right|\right) \\
& \leqslant 2\left(\mathrm{E}\left|h_{1}\left(\bar{S}_{r}\left(K_{0}\left(b_{n}\right)\right)\right) h_{2}\left(\bar{S}_{q}\left(K_{0}\left(b_{n}\right)\right)\right)\right|+\mathrm{E}\left|h_{2}\left(\bar{S}_{r}\left(K_{0}\left(b_{n}\right)\right)\right) h_{1}\left(\bar{S}_{q}\left(K_{0}\left(b_{n}\right)\right)\right)\right|\right. \\
&\left.+\mathrm{E}\left|h_{2}\left(\bar{S}_{r}\left(K_{0}\left(b_{n}\right)\right)\right) h_{2}\left(\bar{S}_{q}\left(K_{0}\left(b_{n}\right)\right)\right)\right|+3\left|T_{n}\right|\left|U_{n}\right|^{-1}\left(\sigma_{r, r} \sigma_{q, q}\right)^{1 / 2}\right) \\
& \leqslant 4\left(\sigma_{r, r} \mathrm{E}\left(\bar{S}_{q}\left(K_{0}\left(b_{n}\right)\right)^{2} \mathbf{1}\left\{\left|\bar{S}_{q}\left(K_{0}\left(b_{n}\right)\right)\right| \geqslant c\right\}\right)\right)^{1 / 2} \\
&+2\left(\sigma_{q, q} \mathrm{E}\left(\bar{S}_{r}\left(K_{0}\left(b_{n}\right)\right)^{2} \mathbf{1}\left\{\left|\bar{S}_{r}\left(K_{0}\left(b_{n}\right)\right)\right| \geqslant c\right\}\right)\right)^{1 / 2}+6\left|T_{n}\right|\left|U_{n}\right|^{-1}\left(\sigma_{r, r} \sigma_{q, q}\right)^{1 / 2},
\end{aligned}
$$

здесь $\mathbf{1}$ - индикатор события.

Нетрудно видеть, что при каждом $r=1, \ldots, k$ семейство $\left\{\bar{S}_{r}^{2}\left(K_{0}\left(b_{n}\right)\right)\right\}_{n=1}^{\infty}$ равномерно интегрируемо. Следовательно, принимая во внимание (30), видим, что для любого $\varepsilon>0$ можно найти $c=c(\varepsilon)$ такое, что при всех достаточно больших $n$

$$
I_{2}^{(1,2)}\left(U_{n}\right)+I_{2}^{(2,1)}\left(U_{n}\right)+I_{2}^{(2,2)}\left(U_{n}\right)<\varepsilon .
$$

Теперь заметим, что

$$
\left(I_{2}^{(1,1)}\left(U_{n}\right)\right)^{2} \leqslant\left|U_{n}\right|^{-2} \sum_{j, t \in U_{n}}\left|\operatorname{cov}\left(h_{1}\left(\bar{S}_{r}\left(Q_{j}\right)\right) h_{1}\left(\bar{S}_{q}\left(Q_{j}\right)\right), h_{1}\left(\bar{S}_{r}\left(Q_{t}\right)\right) h_{1}\left(\bar{S}_{q}\left(Q_{t}\right)\right)\right)\right| .
$$

Учитьвая (32), приходим к неравенствам

$$
\begin{aligned}
& \left|U_{n}\right|^{-2} \sum_{\substack{j, t \in U_{n} \\
\|j-t\| \leqslant 2 b_{n}}}\left|\operatorname{cov}\left(h_{1}\left(\bar{S}_{r}\left(Q_{j}\right)\right) h_{1}\left(\bar{S}_{q}\left(Q_{j}\right)\right), h_{1}\left(\bar{S}_{r}\left(Q_{t}\right)\right) h_{1}\left(\bar{S}_{q}\left(Q_{t}\right)\right)\right)\right| \\
& \leqslant 2 c^{2}\left|U_{n}\right|^{-2} \sum_{\substack{j, t \in U_{n} \\
\|j-t\| \leqslant 2 b_{n}}} \mathrm{E}\left|h_{1}\left(\bar{S}_{r}\left(Q_{j}\right)\right) h_{1}\left(\bar{S}_{q}\left(Q_{j}\right)\right)\right| \\
& \leqslant 2^{d+1} c^{2}\left|U_{n}\right|^{-1}\left|K_{0}\left(b_{n}\right)\right|\left(\sigma_{r, r} \sigma_{q, q}\right)^{1 / 2} .
\end{aligned}
$$


Свойство квази-ассоциированности позволяет получить, что

$$
\begin{gathered}
\left|U_{n}\right|^{-2} \sum_{\substack{j, t \in U_{n} \\
\|j-t\|>2 b_{n}}}\left|\operatorname{cov}\left(h_{1}\left(\bar{S}_{r}\left(Q_{j}\right)\right) h_{1}\left(\bar{S}_{q}\left(Q_{j}\right)\right), h_{1}\left(\bar{S}_{r}\left(Q_{t}\right)\right) h_{1}\left(\bar{S}_{q}\left(Q_{t}\right)\right)\right)\right| \\
\leqslant c^{2}\left|U_{n}\right|^{-2} \sum_{\substack{j, t \in U_{n} \\
\|j-t\|>2 b_{n}}}\left|Q_{j}\right|^{-1 / 2}\left|Q_{t}\right|^{-1 / 2} \sum_{u \in Q_{j}, v \in Q_{t}}\left(\left|\operatorname{cov}\left(X_{u, r}, X_{v, q}\right)\right|\right. \\
\left.\quad+\left|\operatorname{cov}\left(X_{u, q}, X_{v, r}\right)\right|+\left|\operatorname{cov}\left(X_{u, r}, X_{v, r}\right)\right|+\left|\operatorname{cov}\left(X_{u, q}, X_{v, q}\right)\right|\right) .
\end{gathered}
$$

Далее имеем

$$
\begin{aligned}
& \sum_{\substack{j, t \in U_{n} \\
\|j-t\|>2 b_{n}}}\left|Q_{j}\right|^{-1 / 2}\left|Q_{t}\right|^{-1 / 2} \sum_{u \in Q_{j}, v \in Q_{t}}\left|\operatorname{cov}\left(X_{u, r}, X_{v, q}\right)\right| \\
& \leqslant \sum_{u, v \in U_{n}}\left|\operatorname{cov}\left(X_{u, r}, X_{v, q}\right)\right| \sum_{\substack{j \in U_{n} \\
Q_{j} \ni u}}\left|Q_{j}\right|^{-1 / 2} \sum_{\substack{t \in U_{n} \\
Q_{t} \ni v}}\left|Q_{t}\right|^{-1 / 2} \\
& \leqslant \frac{1}{2} \sum_{u, v \in U_{n}}\left|\operatorname{cov}\left(X_{u, r}, X_{v, q}\right)\right|\left[\left(\sum_{\substack{j \in U_{n} \\
Q_{j} \ni u}}\left|Q_{j}\right|^{-1 / 2}\right)^{2}+\left(\sum_{\substack{t \in U_{n} \\
Q_{t} \ni v}}\left|Q_{t}\right|^{-1 / 2}\right)^{2}\right] \\
& \leqslant\left(\left|K_{0}\left(U_{n}\right)\right|\left|U_{n}\right|+\left|K_{0}\left(U_{n}\right)\right|^{2}\left|T_{n}\right|\right) \sigma_{r, q} .
\end{aligned}
$$

Оценивая аналогичным образом все суммы, фигурирующие в правой части неравенства (36), и применяя (35), видим, что

$$
\begin{aligned}
& \left(I_{2}^{(1,1)}\left(U_{n}\right)\right)^{2} \leqslant c^{2}\left\{2^{d+1}\left|U_{n}\right|^{-1}\left|K_{0}\left(b_{n}\right)\right|\left(\sigma_{r, r} \sigma_{q, q}\right)^{1 / 2}\right. \\
& \left.\quad+\left(\left|K_{0}\left(b_{n}\right)\right|\left|U_{n}\right|^{-1}+\left|K_{0}\left(b_{n}\right)\right|^{2}\left|T_{n}\right|\left|U_{n}\right|^{-2}\right)\left(\sigma_{r, q}+\sigma_{q, r}+\sigma_{r, r}+\sigma_{q, q}\right)\right\}
\end{aligned}
$$

Учитьвая (30), (33), (34) и (37), получаем, что

$$
I_{2}\left(U_{n}\right) \rightarrow 0 \quad \text { при } n \rightarrow \infty \text {. }
$$

Заметим, что

$$
\begin{aligned}
\left|U_{n}\right|^{-1} & \sum_{j \in U_{n}}\left|Q_{j}\right|^{-1} \mathrm{E} S_{r}\left(Q_{j}\right) S_{q}\left(Q_{j}\right) \\
= & \left|U_{n}\right|^{-1}\left|U_{n} \backslash T_{n}\right|\left|K_{0}\left(b_{n}\right)\right|^{-1} \mathrm{E} S_{r}\left(K_{0}\left(b_{n}\right)\right) S_{q}\left(K_{0}\left(b_{n}\right)\right) \\
& +\left|U_{n}\right|^{-1} \sum_{j \in T_{n}}\left|Q_{j}\right|^{-1} \mathrm{E} S_{r}\left(Q_{j}\right) S_{q}\left(Q_{j}\right) .
\end{aligned}
$$

В силу (15) справедливо следующее соотношение:

$$
\left|K_{0}\left(b_{n}\right)\right|^{-1} \mathrm{E} S_{r}\left(K_{0}\left(b_{n}\right)\right) S_{q}\left(K_{0}\left(b_{n}\right)\right) \rightarrow c_{r, q} \quad \text { при } n \rightarrow \infty .
$$

Согласно (30) имеем

$$
\left|T_{n}\right|\left|U_{n}\right|^{-1} \rightarrow 0 \quad \text { при } n \rightarrow \infty \text {. }
$$


Воспользовавшись очевидньм неравенством

$$
\left|Q_{j}\right|^{-1} \mathrm{E}\left|S_{r}\left(Q_{j}\right) S_{q}\left(Q_{j}\right)\right| \leqslant\left(\sigma_{r, r} \sigma_{q, q}\right)^{1 / 2}, \quad j \in \mathbb{Z}^{d}
$$

заключаем, что

$$
I_{3}\left(U_{n}\right) \rightarrow 0 \quad \text { при } n \rightarrow \infty \text {. }
$$

Соотношения (31), (38) и (39) влекут (26). Теорема 2 полностью доказана.

Автор признателен доценту С. А. Боровковой и профессору М. Деккингу за приглашение в Технический университет Делфта (Нидерланды), где в декабре 2002 года была выполнена эта работа, а также всем сотрудникам лаборатории CROSS этого университета за гостеприимство.

\section{СПИСОК ЦИТИРОВАННОЙ ЛИТЕРАТУРЫ}

[1] Bulinski A., Suquet C. Normal approximation for quasi-associated random fields // Statist. Probability Lett. 2001. V. 54. P. 215-226.

[2] Булинский А. В. Асимптотическая нормальность квази-ассоциированных векторных случайных полей // Обозрение прикладн. и промьшл. матем. 2000. Т. 7. С. 482-483.

[3] Doukhan P., Louhichi S. A new weak dependence condition and application to moment inequalities // Stoch. Proc. Appl. 1999. V. 84. P. 313-342.

[4] Doukhan P., Lang G. Rates in the empirical central limit theorem for stationary weakly dependent random fields // Statist. Inf. Stoch. Proc. 2002. V. 5. P. 199-228.

[5] Esary J., Proschan F., Walkup D. Association of random variables with applications // Ann. Math. Statist. 1967. V. 38. P. 1466-1474.

[6] Newman C. M. Asymptotic independence and limit theorems for positively and negatively dependent random varialbes // Inequalities in Statistics and Probability / ed. Y.L. Tong. IMS Lecture Notes Monograph Ser. Hayward, CA: Inst. Math. Statist., 1984. P. 127-140.

[7] Joag-Dev K., Proschan F. Negative association of random variables, with applications // Ann. Statist. 1983. V. 11. P. 286-295.

[8] Ebrahimi E. On the dependence structure of certain multidimensional Ito processes and corresponding hitting times // J. Multivar. Anal. 2002. V. 81. P. 128-137.

[9] Evans S. Association and random measures // Probability Theory Related Fields. 1990. V. 86. P. 1-19.

[10] Fortuin C., Kasteleyn P., Ginibre J. Correlation inequalities on some partially ordered sets // Comm. Math. Phys. 1971. V. 22. P. 89-103.

[11] Lee M.-L. T., Rachev S. T., Samorodnitsky G. Association of stable random variables // Ann. Probability. 1990. V. 18. P. 1759-1764.

[12] Newman C. M. Normal fluctuations and FKG-inequalities // Comm. Math. Phys. 1980. V. 74. P. $119-128$.

[13] Pitt L. Positively correlated normal variables are associated // Ann. Probability. 1982. V. 10. P. 496-499.

[14] Rachev S. T., Xin H. Test on association of random variables in the domain of a multivariate stable law // Probability Math. Statist. 1993. V. 14. P. 125-141.

[15] Булинский А. В., Шабанович Э. Асимптотическое поведение некоторых функционалов от положительно и отрицательно зависимых случайных полей // Фундамент. и прикл. матем. 1998. T. 4. C. $479-492$.

[16] Birkel T. On the convergence rate in the central limit theorem for associated processes // Ann. Probab. 1988. V. 16. № 4. P. 1685-1698.

[17] Roussas G. G. Asymptotic normality of random fields of positively and negatively associated processes // J. Multivar. Anal. 1994. V. 50. P. 152-173.

[18] Peligrad M., Shao Q.-M. Estimation of variance of partial sums of an associated sequence of random variables // Stochastic Proc. Appl. 1995. V. 56. P. 307-319. 
[19] Bulinski A. V. On the convergence rates in the central limit theorem for positively and negatively dependent random fields // Proceedings of the Kolmogorov semester in the Euler Math. Inst. (March 1993) / ed. I. A. Ibragimov, A. Yu. Zaitsev. Probability Theory and Mathematical Statistics. London: Gordon and Breach, 1996. P. 3-14.

[20] Burton R., Dabrowski A. R., Dehling H. An invariance principle for quasi-associated random vectors // Stochastic Process. Appl. 1986. V. 23. P. 301-306.

[21] Bulinski A., Shashkin A. Rates in the CLT for vector-valued random fields // Prépublications de Mathématiques de l'Université Paris 10. V. 24. Nanterre, 2003. P. 1-27.

[22] Шашкин А.П. Квази-ассоциированность гауссовских систем векторов // УМН. 2002. T. 57. №6. С. 199-200.

[23] Бахтин Ю. Ю., Булинский А. В. Моментные неравенства для сумм зависимых мультииндексированных величин // Фундамент. и прикл. матем. 1997. Т. 3. С. 1101-1108.

[24] Рюэль Д. Статистическая механика. М.: Мир, 1972.

[25] Bolthausen E. On the central limit theorem for mixing random fields // Ann. Probability. 1982. V. 10. P. 1047-1050.

[26] Булинский А. В., Вронский М. А. Статистический вариант ЦПТ для ассоциированных случайных полей // Фундамент. и прикл. матем. 1996. Т. 2. С. 891-908.

Московский государственный университет им. М.В. Ломоносова

Поступило

E-mail: bulinski@mech.math.msu.su 